\title{
Towards the Albertson conjecture
}

\author{
János Barát* \\ Department of Computer Science and Systems Technology \\ University of Pannonia, Egyetem u. 10, 8200 Veszprém, Hungary \\ barat@dcs.vein.hu \\ Géza Tóth ${ }^{\dagger}$ \\ Rényi Institute, Reáltanoda u. 13-15, 1052 Budapest, Hungary \\ geza@renyi.hu
}

Submitted: Sep 2, 2009; Accepted: May 7, 2010; Published: May 14, 2010

Mathematics Subject Classification: 05C10, 05C15

\begin{abstract}
Albertson conjectured that if a graph $G$ has chromatic number $r$, then the crossing number of $G$ is at least as large as the crossing number of $K_{r}$, the complete graph on $r$ vertices. Albertson, Cranston, and Fox verified the conjecture for $r \leqslant 12$. In this paper we prove it for $r \leqslant 16$.
\end{abstract}

Dedicated to the memory of Michael O. Albertson.

\section{Introduction}

Graphs in this paper are without loops and multiple edges. Every planar graph is fourcolorable by the Four Color Theorem $[2,24]$. The efforts to solve the Four Color Problem had a great effect on the development of graph theory, and FCT is one of the most important theorems of the field.

The crossing number of a graph $G$, denoted $\mathrm{CR}(G)$, is the minimum number of edge crossings in a drawing of $G$ in the plane. It is a natural relaxation of planarity, see [25] for a survey. The chromatic number of a graph $G$, denoted $\chi(G)$, is the minimum number of colors in a proper coloring of $G$. The Four Color Theorem states: if $\operatorname{CR}(G)=0$, then $\chi(G) \leqslant 4$. Oporowski and Zhao [18] proved that every graph with crossing number at most two is 5-colorable. Albertson et al. [5] showed that if $\operatorname{CR}(G) \leqslant 6$, then $\chi(G) \leqslant 6$. It

\footnotetext{
*Research is supported by OTKA Grants PD 75837 and K 76099, and the János Bolyai Research Scholarship of the Hungarian Academy of Sciences.

${ }^{\dagger}$ Research is supported by OTKA T 038397 and T 046246.
} 
was observed by Schaefer that if $\operatorname{CR}(G)=k$, then $\chi(G)=O(\sqrt[4]{k})$, and this is the correct order of magnitude [4].

Graphs with chromatic number $r$ do not necessarily contain $K_{r}$ as a subgraph, they can have clique number 2, see [27]. The Hajós conjecture proposed that graphs with chromatic number $r$ contain a subdivision of $K_{r}$. This conjecture, whose origin is unclear but attributed to Hajós, turned out to be false for $r \geqslant 7$. Also, it was shown by Erdős and Fajtlowicz [9] that almost all graphs are counterexamples. Albertson posed the following

Conjecture 1 If $\chi(G)=r$, then $\mathrm{CR}(G) \geqslant \mathrm{CR}\left(K_{r}\right)$.

This statement is weaker than Hajós' conjecture: if $G$ contains a subdivision of $K_{r}$, then $\mathrm{CR}(G) \geqslant \mathrm{CR}\left(K_{r}\right)$.

For $r=5$, Albertson's conjecture is equivalent to the Four Color Theorem. Oporowski and Zhao [18] verified it for $r=6$. Albertson, Cranston, and Fox [4] proved it for $r \leqslant 12$. In this note, we take one more little step.

Theorem 2 For $r \leqslant 16$, if $\chi(G)=r$, then $\mathrm{CR}(G) \geqslant \mathrm{CR}\left(K_{r}\right)$.

In their proof, Albertson, Cranston and Fox combined lower bounds for the number of edges of $r$-critical graphs, and lower bounds on the crossing number of graphs with given number of vertices and edges. Our proof is very similar, but we use better lower bounds in both cases.

Albertson et al. proved that any minimal counterexample to Conjecture 1 should have less than $4 r$ vertices. We slightly improve this result as follows.

Lemma 3 If $G$ is an $n$-vertex, $r$-critical graph with $n \geqslant 3.57 r$, then $\mathrm{CR}(G) \geqslant \mathrm{CR}\left(K_{r}\right)$.

In Section 2, we review lower bounds for the number of edges of $r$-critical graphs. In Section 3, we discuss lower bounds on the crossing number. In Section 4, we combine these two bounds to obtain the proof of Theorem 2. In Section 5, we prove Lemma 3.

Let $n$ always denote the number of vertices of $G$. In notation and terminology, we follow Bondy and Murty [6]. In particular, the join of two disjoint graphs $G$ and $H$, denoted $G \vee H$, arises by adding all edges between vertices of $G$ and $H$. A vertex $v$ is of full degree, if it has degree $n-1$. If a graph $G$ contains a subdivision of $H$, then $G$ contains a topological $H$. A vertex $v$ is adjacent to a vertex set $X$ means that each vertex of $X$ is adjacent to $v$.

\section{Color-critical graphs}

A graph $G$ is $r$-critical, if $\chi(G)=r$, but all proper subgraphs of $G$ have chromatic number less than $r$. In what follows, let $G$ denote an $r$-critical graph with $n$ vertices and $m$ edges. Since $G$ is $r$-critical, every vertex has degree at least $r-1$, therefore, $2 m \geqslant(r-1) n$. The value $2 m-(r-1) n$ is the excess of $G$. For $r \geqslant 3$, Dirac [7] proved the following: if $G$ is not complete, then $2 m \geqslant(r-1) n+(r-3)$. For $r \geqslant 4$, Dirac [8] gave a characterization 
of $r$-critical graphs with excess $r-3$. For a positive integer $r, r \geqslant 3$, let $\Delta_{r}$ be the following family of graphs. For any graph in the family, let the vertex set consist of three non-empty, pairwise disjoint sets $A, B_{1}, B_{2}$ and two additional vertices $a$ and $b$. Here, $\left|B_{1}\right|+\left|B_{2}\right|=|A|+1=r-1$. The sets $A$ and $B_{1} \cup B_{2}$ both span cliques, $a$ is connected to $A \cup B_{1}$ and $b$ is connected to $A \cup B_{2}$. See Figure 1. Graphs in $\Delta_{r}$ are called Hajós graphs of order $2 r-1$. Observe, that these graphs have chromatic number $r$, and they contain a topological $K_{r}$. Hence they satisfy Hajós' conjecture.

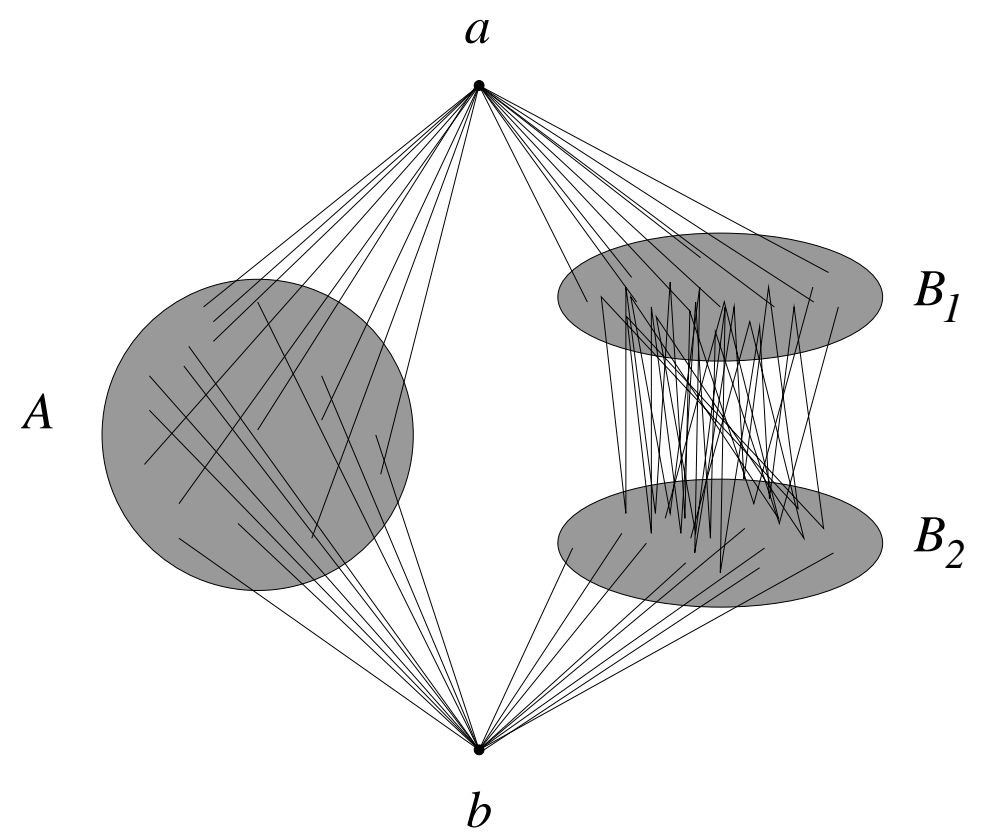

Figure 1: The family $\Delta_{r}$

Gallai [10] proved that any $r$-critical graph with at most $2 r-2$ vertices is the join of two smaller graphs. Therefore, the complement of any such graph is disconnected. Based on this observation, Gallai proved that non-complete $r$-critical graphs on at most $2 r-2$ vertices have much larger excess than in Dirac's result.

Lemma 4 [10] Let $r, p$ be integers, $r \geqslant 4$ and $2 \leqslant p \leqslant r-1$. If $G$ is an $r$-critical graph with $n$ vertices and $m$ edges, where $n=r+p$, then $2 m \geqslant(r-1) n+p(r-p)-2$. Equality holds if and only if $G$ is the join of $K_{r-p-1}$ and $G \in \Delta_{p+1}$.

Since every $G$ in $\Delta_{p+1}$ contains a topological $K_{p+1}$, the join of $K_{r-p-1}$ and $G$ contains a topological $K_{r}$. This yields a slight improvement for our purposes.

Corollary 5 Let $r, p$ be integers, $r \geqslant 4$ and $2 \leqslant p \leqslant r-1$. If $G$ is an $r$-critical graph with $n$ vertices and $m$ edges, where $n=r+p$, and $G$ does not contain a topological $K_{r}$, then $2 m \geqslant(r-1) n+p(r-p)-1$. 
We call the bound given by Corollary 5 the Gallai bound.

For $r \geqslant 3$, let $\mathcal{E}_{r}$ denote the family of the following graphs $G$. The vertex set of any $G$ consists of four non-empty pairwise disjoint sets $A_{1}, A_{2}, B_{1}, B_{2}$, and one additional vertex $c$. Here $\left|B_{1}\right|+\left|B_{2}\right|=\left|A_{1}\right|+\left|A_{2}\right|=r-1$ and $\left|A_{2}\right|+\left|B_{2}\right| \leqslant r-1$. Let $A=A_{1} \cup A_{2}$ and $B=B_{1} \cup B_{2}$. The sets $A$ and $B$ each induce a clique in $G$. The vertex $c$ is connected to $A_{1} \cup B_{1}$. A vertex $a$ in $A$ is adjacent to a vertex $b$ in $B$ if and only if $a \in A_{2}$ and $b \in B_{2}$.

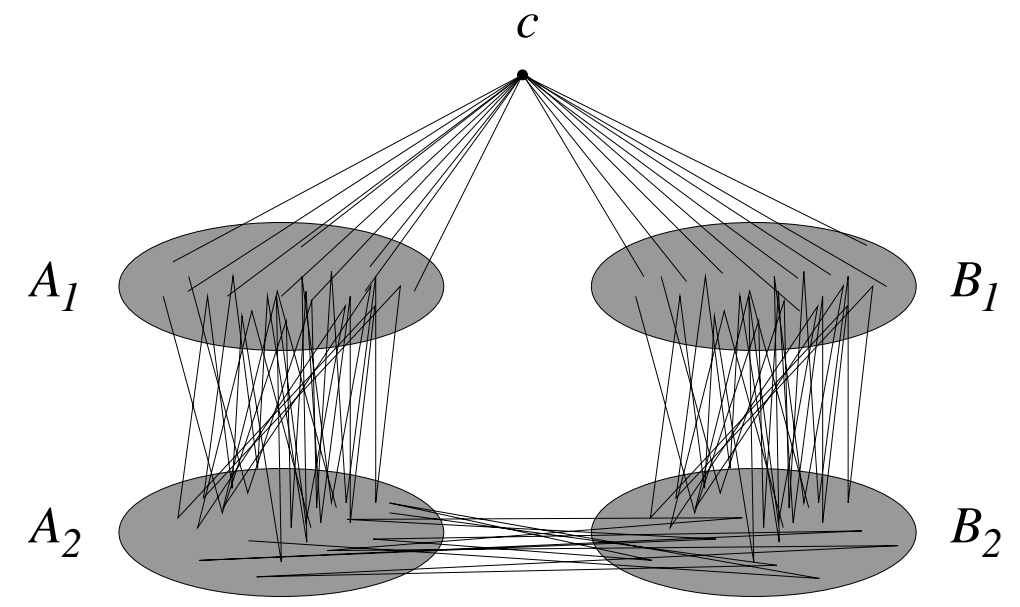

Figure 2: The family $\mathcal{E}_{r}$

Observe, that $\mathcal{E}_{r} \supset \Delta_{r}$, and every graph $G$ in $\mathcal{E}_{r}$ is $r$-critical with $2 r-1$ vertices. Kostochka and Stiebitz [15] improved Dirac's bound as follows.

Lemma 6 [15] Let $r$ be a positive integer, $r \geqslant 4$, and let $G$ be an $r$-critical graph. If $G$ is neither $K_{r}$ nor a member of $\mathcal{E}_{r}$, then $2 m \geqslant(r-1) n+(2 r-6)$.

Corollary 7 Let $r$ be a positive integer, $r \geqslant 4$, and let $G$ be an $r$-critical graph. If $G$ does not contain a topological $K_{r}$, then $2 m \geqslant(r-1) n+(2 r-6)$.

Proof: We show that any member of $\mathcal{E}_{r}$ contains a topological $K_{r}$. The sets $A$ and $B$ both span a complete graph on $r-1$ vertices. We only have to show that vertex $c$ is connected to $A_{2}$ or $B_{2}$ by vertex-disjoint paths. To see this, we observe that $\left|A_{2}\right|$ or $\left|B_{2}\right|$ is the smallest of $\left\{\left|A_{1}\right|,\left|A_{2}\right|,\left|B_{1}\right|,\left|B_{2}\right|\right\}$. Indeed, if $\left|B_{1}\right|$ was the smallest, then $\left|A_{2}\right|>\left|B_{1}\right|$ implies $\left|A_{2}\right|+\left|B_{2}\right|>\left|B_{1}\right|+\left|B_{2}\right|=r-1$ contradicting our assumption. We may assume that $\left|A_{2}\right|$ is the smallest. Now $c$ is adjacent to $A_{1}$, and there is a matching of size $\left|A_{2}\right|$ between $B_{1}$ and $B_{2}$ and between $B_{2}$ and $A_{2}$. Therefore, we can find a set $S$ of disjoint paths from $c$ to $A_{2}$. In this way, $A \cup c \cup S$ is a topological $r$-clique.

The bound in Corollary 7 is the Kostochka, Stiebitz bound, or KS-bound for short.

In what follows, we obtain a complete characterization of $r$-critical graphs on $r+3$ or $r+4$ vertices. 
Lemma 8 For $r \geqslant 8$, there are precisely two $r$-critical graphs on $r+3$ vertices. They can be constructed from two 4-critical graphs on seven vertices by adding vertices of full degree.
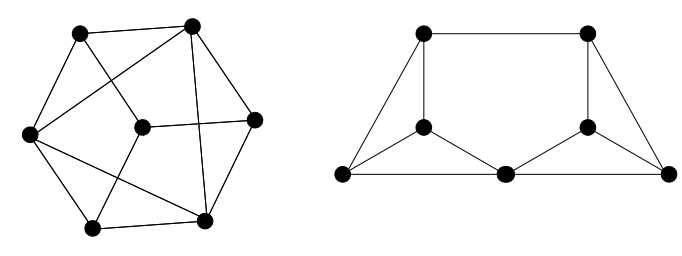

Figure 3: The two 4-critical graphs on seven vertices

PROOF: The proof is by induction on $r$. For the base case $r=8$, there are precisely two 8-critical graphs on 11 vertices, see Royle's complete search [22].

Let $G$ be an $r$-critical graph with $r \geqslant 9$ and $n=r+3 \geqslant 12$. The minimum degree is at least $r-1$, and $r-1=n-4$. If $G$ has a vertex $v$ of full degree, then we use induction. So we may assume that every vertex in $\bar{G}$, the complement of $G$, has degree 1,2 , or 3 . By Gallai's theorem, $\bar{G}$ is disconnected. Observe the following: if there are at least four independent edges in $\bar{G}$, then $\chi(G) \leqslant n-4=r-1$, a contradiction. That is, there are at most three independent edges in $\bar{G}$. Therefore, $\bar{G}$ has two or three components. If there is a triangle in the complement, then we can save two colors. If there were two triangles, then $\chi(G) \leqslant n-4=r-1$, a contradiction.

Assume that there are three components in $\bar{G}$. Since each degree is at least one, there are at least three independent edges. Therefore, there is no triangle in $\bar{G}$ and no path with three edges. That is, the complement consists of three stars. Since the degree is at most three and there are at least 12 vertices, there is only one possibility: $\bar{G}=K_{1,3} \cup K_{1,3} \cup K_{1,3}$, see Figure 4.

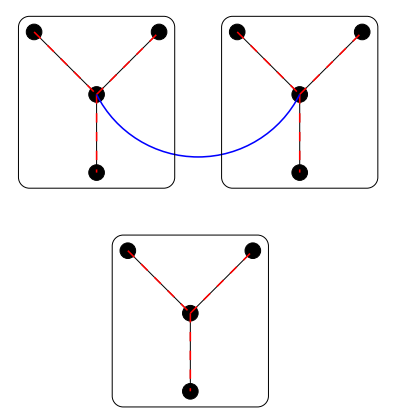

Figure 4: The complement and a removable edge

We have to check whether this concrete graph is indeed critical. Observe, that if we remove the edge connecting two centers of these stars, the chromatic number remains $r$. Therefore, our graph is not $r$-critical, a contradiction. 
In the remaining case, $\bar{G}$ has two components $H_{1}$ and $H_{2}$. Since there are at most three independent edges, there is one in $H_{1}$ and two in $H_{2}$. It implies that $H_{1}$ has at most four vertices. Therefore, $\mathrm{H}_{2}$ has at least eight vertices. Consider a spanning tree $\mathrm{T}$ of $\mathrm{H}_{2}$ and remove two adjacent vertices of $T$, one of them being a leaf. It is easy to see that the remainder of $T$ contains a path with three edges. Therefore, in total we found three independent edges of $\mathrm{H}_{2}$, a contradiction.

In Lemma 10, we characterize $r$-critical graphs on $r+4$ vertices. For that proof, we need the following result of Gallai.

Theorem 9 [10] Let $r \geqslant 3$ and $n<\frac{5}{3} r$. If $G$ is an $r$-critical, $n$-vertex graph, then it contains at least $\left\lceil\frac{3}{2}\left(\frac{5}{3} r-n\right)\right\rceil$ vertices of full degree.

The existence of a vertex of full degree gives rise to an inductive proof of the following

Lemma 10 For $r \geqslant 6$, there are precisely twenty-two $r$-critical graphs on $r+4$ vertices. Each of them can be constructed by adding vertices of full degree to a graph in the following list:

- the 3-critical graph on seven vertices,

- the four 4-critical graphs on eight vertices,

- the sixteen 5-critical graphs on nine vertices, or

- the 6-critical graph on ten vertices.

ProOF: For the base of induction, we use Royle's table again, see [22]. The full computer search shows that there are precisely twenty-two 6-critical graphs on ten vertices. One of them has three vertices of full degree, four of them has two, sixteen graphs have one vertex of full degree, and one graph has no such vertex. For the induction step, we use Theorem 9 , and see that there are at least $r-6$ vertices of full degree. Since $r \geqslant 7$, there is always a vertex of full degree. We remove it, and use the induction hypothesis to finish the proof.

There is an explicit list of twenty-one 5-critical graphs on nine vertices [22]. We had to check that each of those graphs contains a topological $K_{5}$. Mader [16] proved that any $n$-vertex graph with at least $3 n-5$ edges contains the subdivision of $K_{5}$. We made a verification partly manually, partly using Mader's extremal result. Therefore, if we add $r-5$ vertices of full degree to any of these graphs, then the resulting graph contains a topological $K_{r}$. Also, the above mentioned 6-critical graph on ten vertices contains a topological $K_{6}$. These two observations imply the following

Corollary 11 Any $r$-critical graph on at most $r+4$ vertices satisfy the Hajós conjecture.

We believe that 4 can be replaced by any other constant in the above result.

Conjecture 12 For every positive integer $c$, there exists a bound $r(c)$ such that for any $r$, where $r \geqslant r(c)$, any $r$-critical graph on $r+c$ vertices satisfies the Hajós conjecture. 


\section{The crossing number}

It follows from Euler's formula that a planar graph can have at most $3 n-6$ edges. Suppose that $G$ has more than $3 n-6$ edges. By deleting crossing edges one by one, it follows by induction that for $n \geqslant 3$,

$$
\mathrm{CR}(G) \geqslant m-3(n-2)
$$

Pach et al. $[19,21]$ generalized this idea and proved the following lower bounds. Both of them holds for any graph $G$ with $n$ vertices and $m$ edges, $n \geqslant 3$.

$$
\begin{gathered}
\mathrm{CR}(G) \geqslant 7 m / 3-25(n-2) / 3 \\
\mathrm{CR}(G) \geqslant 4 m-103(n-2) / 6 \\
\mathrm{CR}(G) \geqslant 5 m-25(n-2)
\end{gathered}
$$

Inequality (1) is the best for $m \leqslant 4(n-2)$,

(2) is the best for $4(n-2) \leqslant m \leqslant 5.3(n-2)$,

(3) is the best for $5.3(n-2) \leqslant m \leqslant 47(n-2) / 6$,

(4) is the best for $47(n-2) / 6 \leqslant m$.

It was also shown in [19] that (1) can not be improved in the range $m \leqslant 4(n-2)$, and $(2)$ can not be improved in the range $4(n-2) \leqslant m \leqslant 5(n-2)$, apart from an additive constant. Inequalities (3) and (4) are conjectured to be far from optimal. Using the methods in [19], one can obtain an infinite family of such linear inequalities of the form $a m-b(n-2)$. For instance, $\operatorname{CR}(G) \geqslant 3 m-35(n-2) / 3$.

The most important inequality for crossing numbers is undoubtedly the Crossing Lemma, first proved by Ajtai, Chvátal, Newborn, Szemerédi [1], and independently by Leighton [13]. If $G$ has $n$ vertices and $m$ edges, $m \geqslant 4 n$, then

$$
\mathrm{CR}(G) \geqslant \frac{1}{64} \frac{m^{3}}{n^{2}}
$$

The original constant was much larger. The constant $\frac{1}{64}$ comes from the well-known probabilistic proof of Chazelle, Sharir, and Welzl [3]. The basic idea is to take a random induced subgraph and apply inequality (1) for that.

The order of magnitude of this bound can not be improved, see [19]. The best known constant is obtained in [19]. If $G$ has $n$ vertices and $m$ edges, $m \geqslant \frac{103}{16} n$, then

$$
\mathrm{CR}(G) \geqslant \frac{1}{31.1} \frac{m^{3}}{n^{2}}
$$

The proof is very similar to the proof of (5), the main difference is that instead of (1), inequality (3) is applied for the random subgraph. The proof of the following technical lemma is based on the same idea. 
Lemma 13 Suppose that $n \geqslant 10$, and $0<p \leqslant 1$. Let

$$
\mathrm{CR}(n, m, p)=\frac{4 m}{p^{2}}-\frac{103 n}{6 p^{3}}+\frac{103}{3 p^{4}}-\frac{5 n^{2}(1-p)^{n-2}}{p^{4}} .
$$

For any graph $G$ with $n$ vertices and $m$ edges, the following holds:

$$
\mathrm{CR}(G) \geqslant \mathrm{CR}(n, m, p)
$$

Proof: Observe that inequality (3) does not hold for graphs with at most two vertices. For any graph $G$, let

$$
\mathrm{CR}^{\prime}(G)= \begin{cases}\mathrm{CR}(G) & \text { if } n \geqslant 3 \\ 4 & \text { if } n=2 \\ 18 & \text { if } n=1 \\ 35 & \text { if } n=0\end{cases}
$$

It is easy to see that for any graph $G$

$$
\mathrm{CR}^{\prime}(G) \geqslant 4 m-\frac{103}{6}(n-2) .
$$

Let $G$ be a graph with $n$ vertices and $m$ edges. Consider a drawing of $G$ with $\operatorname{CR}(G)$ crossings. Choose each vertex of $G$ independently with probability $p$, and let $G^{\prime}$ be a subgraph of $G$ induced by the selected vertices. Consider the drawing of $G^{\prime}$ inherited from the drawing of $G$. That is, each edge of $G^{\prime}$ is drawn exactly as it is drawn in $G$. Let $n^{\prime}$ and $m^{\prime}$ be the number of vertices and edges of $G^{\prime}$, and let $x$ be the number of crossings in the present drawing of $G^{\prime}$. Notice that $E\left(n^{\prime}\right)=p n, E\left(m^{\prime}\right)=p^{2} m, E(x)=p^{4} \mathrm{CR}(G)$. Using inequality (7), and the linearity of expectations, the following holds:

$$
\begin{aligned}
E(x) & \geqslant E\left(\mathrm{CR}\left(G^{\prime}\right)\right) \geqslant E\left(\mathrm{CR}^{\prime}\left(G^{\prime}\right)\right)-4 P\left(n^{\prime}=2\right)-18 P\left(n^{\prime}=1\right)-35 P\left(n^{\prime}=0\right) \\
& \geqslant 4 p^{2} m-\frac{103}{6} p n+\frac{103}{3}-4\left(\begin{array}{l}
n \\
2
\end{array}\right) p^{2}(1-p)^{n-2}-18 n p(1-p)^{n-1}-35(1-p)^{n} \\
& \geqslant 4 p^{2} m-\frac{103}{6} p n+\frac{103}{3}-5 n^{2}(1-p)^{n-2} .
\end{aligned}
$$

Dividing by $p^{4}$, we obtain the statement of the lemma.

Note that in our applications, $p$ will be at least $1 / 2, n$ will be at least 13 . Therefore, the last term in the inequality, $\frac{5 n^{2}(1-p)^{n-2}}{p^{4}}$, is negligible.

We also need some bounds on the crossing number of the complete graph, $\mathrm{CR}\left(K_{r}\right)$. It is known that

$$
\mathrm{CR}\left(K_{r}\right) \leqslant Z(r)=\frac{1}{4}\left\lfloor\frac{r}{2}\right\rfloor\left\lfloor\frac{r-1}{2}\right\rfloor\left\lfloor\frac{r-2}{2}\right\rfloor\left\lfloor\frac{r-3}{2}\right\rfloor,
$$

see [23]. Guy [11] conjectured $\mathrm{CR}\left(K_{r}\right)=Z(r)$. It has been verified for $r \leqslant 12$, but still open for $r>12$. The best known lower bound is due to de Klerk et al. [14]: $\mathrm{CR}\left(K_{r}\right) \geqslant 0.86 Z(r)$. 


\section{Proof of Theorem 2}

Suppose that $G$ is an $r$-critical graph. If $G$ contains a topological $K_{r}$, then $\operatorname{CR}(G) \geqslant$ $\mathrm{CR}\left(K_{r}\right)$. Suppose in the sequel that $G$ does not contain a topological $K_{r}$. Therefore, we can apply the Kostochka, Stiebitz- and the Gallai bound on the number of edges. Next we use Lemma 13 to get the desired lower bound on the crossing number. Albertson et al. used the same approach in [4]. They used a weaker version of the bounds, and instead of Lemma 13, they applied the weaker inequality (3). In the tables below, we include the results of our calculations. For comparison, we also include the result Albertson et al. might have had using (3). In the appendix, we present our simple Maple program performing all calculations.

1. Let $r=13$. By $(8)$, we have $\mathrm{CR}\left(K_{13}\right) \leqslant 225$. By Corollary 11 , we only need to consider $n \geqslant r+5=18$. If $n \geqslant 22$, then the KS-bound combined with (3) gives the desired result: $2 m \geqslant 12 n+20 \Rightarrow \mathrm{CR}(G) \geqslant 4(6 n+10)-103 / 6(n-2) \geqslant 224.67$.

For $18 \leqslant n \leqslant 21$ the result follows from the table below.

\begin{tabular}{|c|c|c|c|c|}
\hline$n$ & $m$ & bound $(3)$ & $p$ & $\lceil\mathrm{CR}(n, m, p)\rceil$ \\
\hline 18 & 128 & 238 & 0.719 & 288 \\
19 & 135 & 249 & 0.732 & 296 \\
20 & 141 & 255 & 0.751 & 298 \\
21 & 146 & 258 & 0.774 & 294 \\
\hline
\end{tabular}

2. Let $r=14$. By $(8)$, we have $\operatorname{CR}\left(K_{14}\right) \leqslant 315$. By Corollary 11 , we only need to consider $n \geqslant r+5=19$. If $n \geqslant 27$, then the KS-bound combined with (3) gives the desired result: $2 m \geqslant 13 n+22 \Rightarrow \mathrm{CR}(G) \geqslant 4(6.5 n+11)-103 / 6(n-2) \geqslant 316$.

For $19 \leqslant n \leqslant 26$ the result follows from the table below.

\begin{tabular}{|c|c|c|c|c|}
\hline$n$ & $m$ & bound $(3)$ & $p$ & $\lceil\mathrm{CR}(n, m, p)\rceil$ \\
\hline 19 & 146 & 293 & 0.659 & 388 \\
20 & 154 & 307 & 0.670 & 402 \\
21 & 161 & 318 & 0.684 & 407 \\
22 & 167 & 325 & 0.702 & 406 \\
23 & 172 & 328 & 0.723 & 398 \\
24 & 176 & 327 & 0.747 & 384 \\
25 & 179 & 322 & 0.775 & 366 \\
26 & 181 & 312 & 0.807 & 344 \\
\hline
\end{tabular}

3. Let $r=15$. By $(8)$, we have $\mathrm{CR}\left(K_{15}\right) \leqslant 441$. By Corollary 11 , we only need to consider $n \geqslant r+5=20$. Suppose now that $G$ is 15 -critical and $n \geqslant 28$. By the KS-bound we have $m \geqslant 7 n+12$. Apply Lemma 13 with $p=0.764$ and a straightforward calculation gives $\mathrm{CR}(G) \geqslant \mathrm{CR}(n, m, 0.764) \geqslant 441$.

For $20 \leqslant n \leqslant 27$ the result follows from the table below. 


\begin{tabular}{|c|c|c|c|c|}
\hline$n$ & $m$ & bound $(3)$ & $p$ & $\lceil\mathrm{CR}(n, m, p)\rceil$ \\
\hline 20 & 165 & 351 & 0.610 & 510 \\
21 & 174 & 370 & 0.617 & 531 \\
22 & 182 & 385 & 0.623 & 542 \\
23 & 189 & 396 & 0.642 & 545 \\
24 & 195 & 403 & 0.659 & 539 \\
25 & 200 & 406 & 0.678 & 526 \\
26 & 204 & 404 & 0.700 & 508 \\
27 & 207 & 399 & 0.725 & 484 \\
\hline
\end{tabular}

4. Let $r=16$. By $(8)$, we have $\mathrm{CR}\left(K_{16}\right) \leqslant 588$. By Corollary 11 , we only need to consider $n \geqslant r+5=21$. Suppose now that $G$ is 16 -critical and $n \geqslant 32$. By the KS-bound we have $m \geqslant 7.5 n+13$. Apply Lemma 13 with $p=0.72$ and again a straightforward calculation gives $\mathrm{CR}(G) \geqslant \mathrm{CR}(n, m, 0.72) \geqslant 588$.

For $21 \leqslant n \leqslant 31$ the result follows from the table below.

\begin{tabular}{|c|c|c|c|c|}
\hline$n$ & $m$ & bound $(4)$ & $p$ & $\lceil\mathrm{CR}(n, m, p)\rceil$ \\
\hline 21 & 185 & 450 & 0.567 & 657 \\
22 & 195 & 475 & 0.573 & 687 \\
23 & 204 & 495 & 0.581 & 706 \\
24 & 212 & 510 & 0.592 & 714 \\
25 & 219 & 520 & 0.605 & 712 \\
26 & 225 & 525 & 0.621 & 701 \\
27 & 230 & 525 & 0.639 & 683 \\
28 & 234 & 520 & 0.659 & 658 \\
29 & 237 & 510 & 0.681 & 628 \\
30 & 239 & 495 & 0.706 & 593 \\
31 & 246 & 505 & 0.713 & 601 \\
\hline
\end{tabular}

This concludes the proof of Theorem 2 .

Remark For $r \geqslant 17$, we could not completely verify Albertson's conjecture. By (8), $\mathrm{CR}\left(K_{17}\right) \leqslant 784$. By Corollary 11 , we only need to consider $n \geqslant r+5=22$.

Lemma 14 Let $G$ be a 17 -critical graph on $n$ vertices. If $n \geqslant 35$, then $\mathrm{CR}(G) \geqslant 784 \geqslant$ $\mathrm{CR}\left(K_{17}\right)$.

ProOF: Let $p=0.681$. Then $\operatorname{CR}(G) \geqslant \operatorname{CR}(n, m, 0.681) \geqslant 14.64 n+280.38$. Therefore, if $n \geqslant \frac{784-280.38}{14.64} \geqslant 34.4$, then we are done.

The next table contains our calculations. There are three cases, $n=32,33,34$, for which our approach is not sufficient. 


\begin{tabular}{|c|c|c|c|c|}
\hline$n$ & $m$ & bound $(4)$ & $p$ & $\lceil\mathrm{CR}(n, m, p)\rceil$ \\
\hline 22 & 206 & 530 & 0.530 & 832 \\
23 & 217 & 560 & 0.534 & 874 \\
24 & 227 & 585 & 0.541 & 902 \\
25 & 236 & 605 & 0.550 & 917 \\
26 & 244 & 620 & 0.560 & 920 \\
27 & 251 & 630 & 0.573 & 913 \\
28 & 257 & 635 & 0.588 & 897 \\
29 & 262 & 635 & 0.604 & 872 \\
30 & 266 & 630 & 0.622 & 840 \\
31 & 269 & 620 & 0.643 & 802 \\
\hline 32 & 271 & 605 & 0.665 & 759 \\
33 & 278 & 615 & 0.672 & 765 \\
34 & 286 & 630 & 0.677 & 779 \\
\hline
\end{tabular}

Lemma 15 Let $G$ be a 17 -critical graph on 32 vertices. Then $\mathrm{CR}(G) \geqslant \mathrm{CR}\left(K_{17}\right)$.

PROOF: Gallai [10] proved that any $r$-critical graph on at most $2 r-2$ vertices is a join of two smaller critical graphs. In our case, $r=17$, and $n=2 r-2=32$. Assume that $G=G_{1} \vee G_{2}$, where $G_{1}$ is $r_{1}$-critical on $n_{1}$ vertices, $G_{2}$ is $r_{2}$-critical on $n_{2}$ vertices, where $17=r_{1}+r_{2}$ and $32=n_{1}+n_{2}$. The sum of the degrees of $G$ can be expressed as the sum of the degrees of the vertices in $G_{i}$, for $i=1,2$, plus twice the number of edges between $G_{1}$ and $G_{2}$ :

$$
2 m \geqslant\left(r_{1}-1\right) n_{1}+\left(r_{2}-1\right) n_{2}+2(r-6)+2 n_{1} n_{2} .
$$

Here, we used the KS-bound for the smaller parts, $G_{1}, G_{2}$. The right-hand side is minimal, if $r_{1} n_{1}+r_{2} n_{2}+2 n_{1} n_{2}$ is minimal. With equivalent modifications, we get the following: $n_{1}\left(r_{1}+n_{2}\right)+n_{2}\left(r_{2}+n_{1}\right)=n_{1}\left(r_{1}+n-n_{1}\right)+\left(n-n_{1}\right)\left(r-r_{1}+n_{1}\right)=\left(n_{1}-r_{1}\right)\left(n-2 n_{1}\right)+$ $n r+n_{1}(n-r)$. This expression is minimal, if $n_{1}$ is minimal and $n_{1}=r_{1}$. This yields the following: $2 m \geqslant n(r-1)+2 n-r-n+2(r-6)$. In our case, it yields $m \geqslant 275$. Next we apply Lemma 13 with $p=0.665$, and we get $\mathrm{CR}(32,275,0.665) \geqslant 795>\mathrm{CR}\left(K_{17}\right)$.

\section{$5 \quad$ Proof of Lemma 3}

Suppose that $r \geqslant 17$, and $G$ is an $r$-critical graph with $n$ vertices and $m$ edges. If $n \geqslant 4 r$, then the statement of the lemma holds by [4]. Suppose that $n=\alpha r$ and $3.57 \leqslant \alpha \leqslant 4$. In order to estimate the crossing number of $G$, instead of the probabilistic argument in the proof of Lemma 13, we apply inequality (3) for each induced subgraph of $G$ with exactly 52 vertices. Let $k=\left(\begin{array}{c}n \\ 52\end{array}\right)$, and let $G_{1}, G_{2}, \ldots, G_{k}$ be the induced subgraphs of $G$ with 52 vertices. Suppose that $G_{i}$ has $m_{i}$ edges. By (3), the following holds for any $i$ :

$$
\mathrm{CR}\left(G_{i}\right) \geqslant 4 m_{i}-\frac{103}{6} \cdot 50
$$


consequently,

$$
\begin{aligned}
\mathrm{CR}(G) & \geqslant \frac{1}{\left(\begin{array}{c}
n-4 \\
48
\end{array}\right)} \sum_{i=1}^{k}\left(4 m_{i}-\frac{103}{6} \cdot 50\right)=\frac{4 m}{\left(\begin{array}{c}
n-4 \\
48
\end{array}\right)}\left(\begin{array}{c}
n-2 \\
50
\end{array}\right)-\frac{50}{\left(\begin{array}{c}
n-4 \\
48
\end{array}\right)} \frac{103}{6}\left(\begin{array}{c}
n \\
52
\end{array}\right) \\
& =\frac{4(n-2)(n-3) m}{50 \cdot 49}-\frac{103}{6} \frac{n(n-1)(n-2)(n-3)}{52 \cdot 51 \cdot 49} \\
& \geqslant \frac{2(n-2)(n-3) n(r-1)}{50 \cdot 49}-\frac{103}{6} \frac{n(n-1)(n-2)(n-3)}{52 \cdot 51 \cdot 49} \\
& =\frac{n(n-2)(n-3)}{49}\left(\frac{r-1}{25}-\frac{103(n-1)}{6 \cdot 52 \cdot 51}\right)
\end{aligned}
$$

since we counted each possible crossing at most $\left(\begin{array}{c}n-4 \\ 48\end{array}\right)$ times, and each edge of $G$ exactly $\left(\begin{array}{c}n-2 \\ 50\end{array}\right)$ times. Finally, some calculation shows that this lower bound is greater than

$$
\frac{r(r-2)(r-3)}{49}(r-1)\left(\frac{1}{25}-\frac{103 \alpha}{6 \cdot 52 \cdot 51}\right) \geqslant \frac{1}{64} r(r-1)(r-2)(r-3)>\mathrm{CR}\left(K_{r}\right)
$$

for $3.57 \leqslant \alpha \leqslant 4$, which proves the lemma.

\section{Remarks}

1. As we have already mentioned, see (6), the best known constant in the Crossing Lemma, 1/31.1, is obtained in [19]. Montaron [17] managed to improve it slightly for dense graphs, that is, in the case when $m=O\left(n^{2}\right)$. His calculations are similar to the proof of Lemma 3 and 13.

2. Our attack of the Albertson conjecture is based on the following philosophy. We calculate a lower bound for the number of edges of an $r$-critical $n$-vertex graph $G$. Next we substitute this into the lower bound given by Lemma 13. Finally, we compare the result and $Z(r)$. For large $r$, this method is not sufficient, but it gives the right order of magnitude, and the constants are roughly within a factor of 4 .

Let $G$ be an $r$-critical graph with $n$ vertices, where $r \leqslant n \leqslant 3.57 r$. Then $2 m \geqslant(r-1) n$. We can apply (6):

$$
\mathrm{CR}(G) \geqslant \frac{1}{31.1} \frac{((r-1) n / 2)^{3}}{n^{2}}=\frac{(r-1)^{3} n}{31.1 \cdot 8} \geqslant \frac{1}{250} r(r-1)^{3} \geqslant \frac{Z(r)}{4} .
$$

3. Let $G=G(n, p)$ be a random graph with $n$ vertices and edge probability $p=p(n)$. It is known [12], that there exists a constant $C_{0}>0$ such that if $n p>C_{0}$, then asymptotically almost surely

$$
\chi(G)<\frac{n p}{\log n p} .
$$

Therefore, asymptotically almost surely

$$
\mathrm{CR}\left(K_{\chi(G)}\right) \leqslant Z(\chi(G))<\frac{n^{4} p^{4}}{64 \log ^{4} n p} .
$$


On the other hand, by [20], if $n p>20$, then almost surely

$$
\mathrm{CR}(G) \geqslant \frac{n^{4} p^{2}}{20000}
$$

Consequently, almost surely we have $\mathrm{CR}(G)>\mathrm{CR}\left(K_{\chi(G)}\right)$. Roughly speaking, unlike in the case of the Hajós conjecture, a random graph almost surely satisfies the statement of the Albertson conjecture.

4. If we do not believe in Albertson's conjecture, we have to look for a counterexample in the range $n \leqslant 3.57 r$. Any candidate must also be a counterexample for the Hajós Conjecture. It is tempting to look at Catlin's graphs.

Let $C_{5}^{k}$ denote the graph arising from $C_{5}$ by repeating each vertex $k$ times. That is, each vertex of $C_{5}$ is blown up to a complete graph on $k$ vertices, and any edge of $C_{5}$ is blown up to a complete bipartite graph $K_{k, k}$.

Lemma 16 Catlin's graphs satisfy the Albertson conjecture.

ProOF: It is known that $\chi\left(C_{5}^{k}\right)=\left\lceil\frac{5}{2} k\right\rceil$. To draw $C_{5}^{k}$, we must draw two copies of $K_{2 k}$, a $K_{k}$ and three copies of $K_{k, k}$. Therefore,

$$
\operatorname{cr}\left(C_{5}^{k}\right) \geqslant 2 Z(2 k)+Z(k)+3 c r\left(K_{k, k}\right) \sim 2 \frac{1}{4} k^{4}+\frac{1}{4}\left(\frac{k}{2}\right)^{4}+3\left(\frac{k}{2}\right)^{4}>0.70 k^{4} .
$$

On the other hand

$$
\operatorname{cr}\left(K_{\chi\left(C_{5}^{k}\right)}\right) \sim \operatorname{cr}\left(K_{\frac{5}{2} k}\right) \leqslant \frac{1}{4}\left(\frac{5}{4} k\right)^{4}<0.62 k^{4}
$$

which proves the claim.

Acknowledgement. We are very grateful to the anonymous referee for his comments.

\section{References}

[1] M. Ajtai, V. Chvátal, M. Newborn, and E. Szemerédi, Crossing-free subgraphs, Annals of Discrete Mathematics 12 (1982), 9-12.

[2] K. Appel and W. Haken, Every planar map is four colorable, Part I. Discharging, Illinois J. Math. 21 (1977), 429-490.

[3] M. Aigner and G. Ziegler, Proofs from the Book, Springer-Verlag, Heidelberg, (2004), viii+239 pp.

[4] M.O. Albertson, D.W. Cranston and J. Fox, Crossings, Colorings and Cliques, Electron. J. Combin., 16 (2009), \#R45.

[5] M.O. Albertson, M. Heenehan, A. McDonough, and J. Wise, Coloring graphs with given crossing patterns, (manuscript). 
[6] A. Bondy and U.S.R. Murty, Graph Theory, Graduate Texts in Mathematics, 244. Springer, New York, (2008) xii+651 pp.

[7] G.A. Dirac, A theorem of R.L. Brooks and a conjecture of H. Hadwiger, Proc. London Math. Soc. 7 (1957), 161-195.

[8] G.A. Dirac, The number of edges in critical graphs, J. Reine Angew. Math. 268/269 (1974), 150-164.

[9] P. Erdős, S. Fajtlowicz, On the conjecture of Hajós. Combinatorica 1 (1981), 141-143.

[10] T. Gallai, Kritische Graphen. II. (German), Magyar Tud. Akad. Mat. Kutat Int. Közl., 8 (1963), 373-395.

[11] R.K. Guy, Crossing numbers of graphs, in: Graph theory and applications (Proc. Conf. Western Michigan Univ., Kalamazoo, Mich., 1972) Lecture Notes in Mathematics 303, Springer, Berlin, 111-124.

[12] S. Janson, T. Łuczak, A. Ruciński: Random Graphs, Wiley, 2000.

[13] T. Leighton, Complexity Issues in VLSI, in: Foundations of Computing Series, MIT Press, Cambridge, MA, (1983).

[14] E. de Klerk, J. Maharry, D.V. Pasechnik, R.B. Richter, G. Salazar, Improved bounds for the crossing numbers of $K_{m, n}$ and $K_{n}$, SIAM J. Discrete Math. 20 (2006), 189202.

[15] A.V. Kostochka and M. Stiebitz, Excess in colour-critical graphs, Graph theory and combinatorial biology (Balatonlelle, 1996), 87-99, Bolyai Soc. Math. Stud., 7, János Bolyai Math. Soc., Budapest, (1999).

[16] W. Mader, $3 n-5$ edges do force a subdivision of $K_{5}$, Combinatorica 18 (1998), 569-595.

[17] B. Montaron, An improvement of the crossing number bound. J. Graph Theory 50 (2005), 43-54.

[18] B. Oporowski, D. Zhao, Coloring graphs with crossings, Discrete Math. 309 (2009), 2948-2951.

[19] J. Pach, R. Radoičić, G. Tardos, G. Tóth, Improving the crossing lemma by finding more crossings in sparse graphs, Discrete Comput. Geom. 36 (2006), 527-552.

[20] J. Pach, G. Tóth: Thirteen problems on crossing numbers, Geombinatorics 9 (2000), 194-207.

[21] J. Pach, G. Tóth: Graphs drawn with few crossings per edge, Lecture Notes in Computer Science 1190, Springer-Verlag, 1997, 345-354. Also in: Combinatorica 17 (1997), 427-439.

[22] Gordon Royle's small graphs. http://units.maths.uwa.edu.au/ gordon/remote/graphs/

[23] B. Richter and C. Thomassen, Relations between crossing numbers of complete and complete bipartite graphs, Amer. Math. Monthly 104 (1997), 131-137. 
[24] N. Robertson, D.P. Sanders, P.D. Seymour, and R. Thomas, The four-color theorem, J. Combin. Theory Ser. B $\mathbf{7 0}$ (1997), 2-44.

[25] L. Székely, A successful concept for measuring non-planarity of graphs: the crossing number, Discrete Math. 276 (2004), 331-352.

[26] K. Zarankiewicz, On a problem of P. Turán concerning graphs. Fund. Math. 41 (1954), 137-145.

[27] A. A. Zykov, On some properties of linear complexes (in Russian) Mat. Sbornik N. S. 24 (1949), 163-188. Reprinted: Translations Series 1, Algebraic Topology (1962), 418-449, AMS, Providence.

\section{Appendix}

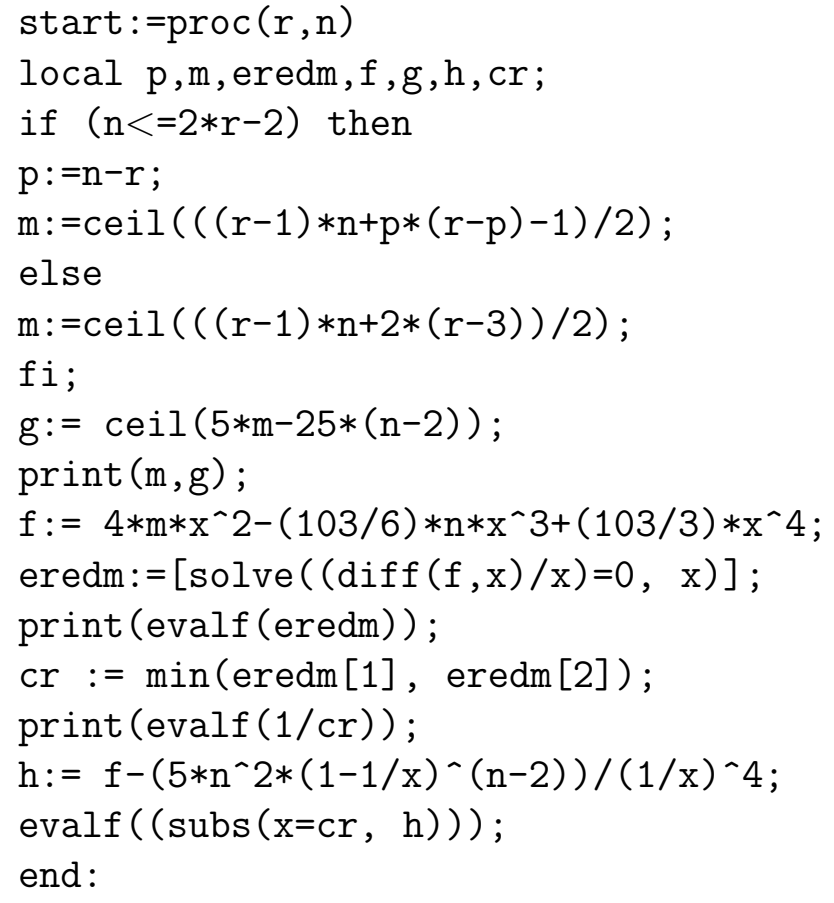

